\title{
Relationship of Post-Stroke Aphasic Types with Sex, Age and Stroke Types
}

\author{
Jingfan Yao1,2,3,4*, Zaizhu Han ${ }^{*}$, Yanli Song1,2,3,4, Lei Li1,2,3,4, Yun Zhou1,2,3,4, \\ Weikang Chen6, Yongmei Deng1,2,3,4, Yongjun Wang1,2,3,4, Yumei Zhang1,2,3,4\# \\ ${ }^{1}$ Department of Neurology, Beijing Tiantan Hospital, Capital Medical University, Beijing, China \\ ${ }^{2}$ China National Clinical Research Center for Neurological Diseases, Beijing, China \\ ${ }^{3}$ Center of Stroke, Beijing Institute for Brain Disorders, Beijing, China \\ ${ }^{4}$ Beijing Key Laboratory of Translational Medicine for Cerebrovascular Disease, Beijing, China \\ ${ }^{5}$ State Key Laboratory of Cognitive Neuroscience and Learning and IDG/McGovern Institute for Brain Research, \\ Beijing Normal University, Beijing, China \\ ${ }^{6}$ The Sixth Affiliated Hospital of Wenzhou Medical University Lishui City People's Hospital, Lishui, China \\ Email: "zhangyumei95@aliyun.com
}

Received 3 January 2015; accepted 25 January 2015; published 28 January 2015

Copyright (C) 2015 by authors and Scientific Research Publishing Inc.

This work is licensed under the Creative Commons Attribution International License (CC BY).

http://creativecommons.org/licenses/by/4.0/

c) (i) Open Access

\section{Abstract}

Aim: To explore what is the relationship of the types of post-stroke aphasia with sex, age and stroke types. Methods: Retrospective analysis was administrated on data of 421 patients with acute stroke. Western battery aphasia was used to measure aphasiac type and aphasia quotient (AQ) score. The patients were divided into three age groups: young, middle-aged and elderly. The stroke types were classified into cerebral infraction (CI) and intracerebral hemorrhage (ICH). Results: All subjects were right-handed, which males and females accounted for $69.60 \%$ and $30.40 \%$, respectively. There were 116 cases of Broca's aphasia (85 males), 35 cases of Wernicke's aphasia (20 males), 15 cases of conductive aphasia (10 males), 63 cases of transcortical motor aphasia (50 males), 11 cases of transcortical sensory aphasia (8 males), 27 cases of transcortical combined aphasia (13 males), 73 cases of anomic aphasia ( 47 males) and 81 cases of global aphasia (60 males). Male patients $(69.60 \%)$ have a significantly higher morbidity of aphasia than that of females $(30.40 \%)$ after stroke $\left(\chi^{2}=11.57, P=0.003\right)$, especially those under 65 years old $(73.38 \%)$. For people 65 years and older, the morbidity of female $(42.97 \%)$ tends to increase with age. Sex has no significant influence on the types of aphasia $\left(\chi^{2}=13.84, P=0.054\right)$. Broca's aphasia is the most common type in both male and female $(29.01 \%, 24.22 \%$, respectively). The distribution of aphasic types has no obvious difference among three age groups $\left(\chi^{2}=14.94, P=0.382\right)$. Aphasia induced by CI (306 cases) is more common than that by ICH (115 cases), but there was no difference in distribution of types of aphasia $\left(\chi^{2}=13.23, P=0.067\right)$. Conclusions: Male patients have a significantly higher level of morbidity of aphasia than females after stroke and a lower average age of onset than females. Broca's

\footnotetext{
*These authors are equal to the article.

${ }^{\#}$ Corresponding author.
}

How to cite this paper: Yao, J.F., Han, Z.Z., Song, Y.L., Li, L., Zhou, Y., Chen, W.K., Deng, Y.M., Wang, Y.J. and Zhang, Y.M. (2015) Relationship of Post-Stroke Aphasic Types with Sex, Age and Stroke Types. World Journal of Neuroscience, 5, 34-39. 
aphasia is the most common one in both male and female. Broca's aphasia, global and anomic aphasia are the most common aphasic types in both $\mathrm{CI}$ and ICH patients, except the female with ICH.

\section{Keywords}

Type of Aphasia, Sex, Age, Type of Stroke

\section{Introduction}

About 82.37\% of stroke patients suffer from speech disorder [1]. The patients of aphasia, as one kind of speech disorder, have a prevalence of 30.25\% - 42.4\% [1]-[4] in stroke patients. Aphasia has heavily influenced the life quality of patients and is one of the most important indicators to evaluate the patients' social outcomes [5]. Previous studies [6]-[10] have shown that many factors can affect the types of aphasia, such as lesion location, types of stroke, age, sex, and intervals between the onset and examination. This study aims to further analyze the distribution of aphasia types in post-stroke aphasia patients and explore the relationship of the types of aphasia with sex, age and stroke types.

\section{Materials and Methods}

\subsection{Participants}

We recruited 421 patients with acute stroke from Department of Neurology of Beijing Tiantan Hospital, Capital Medical University from July 2005 to July 2012. They met the inclusion and exclusion criteria (see below). They were divided into three groups of different ages: youth one ( $\leq 40 \mathrm{y})$, middle-aged one (41 - $65 \mathrm{y}$ ) and elderly one (>65 y).

\subsection{Inclusion Criteria}

1) The diagnosis of stroke accorded with the criteria [11] of the Fourth National Cerebralvascular Conference and was confirmed by the computed tomography (CT) or magnetic resonance imaging (MRI); 2) The patients were from inpatients of neurology department; 3) Their native language was Chinese language; 4) Patients had clear consciousness and could cooperate; 5) The degree of formal education was above the elementary school, intelligence was normal prior to the onset of stroke, and there was no the history of mental illness; 6) There was no speech disorder before the onset, whether the stroke was Initial or repeated; 7) Aphasia quotient (AQ) scores calculated by the Western battery aphasia were less than 93.8 .

\subsection{Exclusion Criteria}

1) Patients had speech disorders, while CT or MRI images could not support the diagnosis of cerebral infraction or intracerebral hemorrhage; 2) They had a consciousness disturbance and could not cooperate; 3) They had speech disorders before the onset of stroke, or combined with other diseases which could affect the speech; 4) They combined with cognitive dysfunction such as dementia and mental disorder; 5) They combined with severe liver, kidney or other medical diseases and audio-visual and comprehensive difficulties; 6) His or her AQ scores was more than 93.8 .

\subsection{Handedness Judgment}

Patients' handedness was evaluated following the criteria of handedness of Aphasia Battery of Chinese instituted by Department of Neurology Peking University First Hospital [12].

\subsection{Aphasic Type Evaluation}

The aphasic type of each patient was assessed by professional language therapist, using Western Battery Aphasia Test. It was administrated within three weeks after recruitment. 


\subsection{Imaging Data Collection}

Every patient was collected CT or/and MRI imaging data.

\subsection{Statistical Analysis}

SPSS 19.0 software package was used to analyze this set of data. The independent sample $t$-test was for the intergroup comparison and chi-square test for the intergroup comparison. The threshold with $P<0.05$ was implemented.

\section{Results}

\subsection{Basic Information}

Four hundred and twenty-one patients (293 males) met the inclusion and exclusion criteria. The mean age was 56.58 years of all patients ( $S D=13.94$, range: $15-87), 55.28$ years of male ones $(S D=13.32$, range: 15 - 87) and 59.54 years of female ones ( $S D=14.90$, range: 21 - 87). The mean age of stroke onset in male patients was significantly higher than female ones $(F=2.562, t=-2.907, P=0.004, \alpha=0.05)$.

\subsection{Handedness}

All the 421 patients were right-handed.

\subsection{Types of Aphasia}

Western Battery Aphasia Test revealed that our patients showed eight types of aphasia (Table 1). They were Broca's aphasia: 116 patients (85 males), Wernicke’s aphasia: 35 patients (20 males), conductive aphasia: 15 patients (10 males), transcortical motor aphasia: 63 patients (50 males), transcortical sensory aphasia: 11 patients (8 males), transcortical combined aphasia: 27 patients (13 males), anomic aphasia: 73 patients (47 males) and global aphasia: 81 patients (60 males).

Table 1 shows that the incidence rate of post-stroke aphasia in the middle-aged and elderly population was higher than that in the youth population (55.82\%, 31.35\%, 12.83\% respectively). Broca's aphasia was the most common type in the three age groups, followed by global and anomic aphasia. Distribution of aphasic types was equivalent among three age groups $\left(\chi^{2}=14.94, P=0.382, \alpha=0.05\right)$. In other words, age had no significant effect on the types of aphasia.

Male patients had a significantly higher level of morbidity of aphasia than that of females after stroke $\left(\chi^{2}=\right.$ 11.57, $P=0.003, \alpha=0.05$ ). After 65 years old, the morbidity of female tended to increase with age (42.97\%), and the gap of morbidity between males and females diminished (26.28\%). Sex had no significant influence on the types of aphasia $\left(\chi^{2}=13.84, P=0.054, \alpha=0.05\right)$. Broca's aphasia was the most common type in both male and female $(29.01 \%, 24.22 \%$, respectively). Global, Wernicke's and anomic aphasia were also common, but transcortical sensory aphasia was the most rare.

Table 1. The distribution of the types of aphasia in different age and sex groups [n (\%)].

\begin{tabular}{|c|c|c|c|c|c|c|c|}
\hline \multirow{2}{*}{ Types of aphasia } & \multicolumn{3}{|c|}{ Male } & \multicolumn{3}{|c|}{ Female } & \multirow{2}{*}{ Total } \\
\hline & Youth & Middle-aged & Elderly & Youth & Middle-aged & Elderly & \\
\hline Broca's & $14(4.78)$ & $51(17.41)$ & $20(6.83)$ & $2(1.56)$ & 14 (10.94) & 15 (11.72) & $116(27.55)$ \\
\hline Wernicke's & $4(1.37)$ & $11(3.75)$ & $5(1.71)$ & $2(1.56)$ & $8(6.25)$ & $5(3.91)$ & $35(8.31)$ \\
\hline Conductive & $3(1.02)$ & 4 (1.37) & $3(1.02)$ & $1(0.78)$ & 4 (3.13) & $0(0.00)$ & $15(3.56)$ \\
\hline Transcortical sensory & $2(0.68)$ & $2(0.68)$ & $4(1.37)$ & $1(0.78)$ & $1(0.78)$ & $1(0.78)$ & $11(2.61)$ \\
\hline Transcortical motor & $2(0.68)$ & 36 (12.29) & $12(4.10)$ & $1(0.78)$ & $7(5.47)$ & $5(3.91)$ & $63(14.96)$ \\
\hline Transcortical combined & $0(0.00)$ & $10(3.41)$ & $3(1.02)$ & $2(1.56)$ & $5(3.91)$ & $7(5.47)$ & $27(6.41)$ \\
\hline Global & $8(2.73)$ & 34 (11.60) & $18(6.14)$ & $1(0.78)$ & $10(7.81)$ & $10(7.81)$ & $81(19.24)$ \\
\hline Anomic & $8(2.73)$ & $27(9.22)$ & $12(4.10)$ & $3(2.34)$ & 11 (8.59) & $12(9.38)$ & $73(17.34)$ \\
\hline Total & & $293(69.60)$ & & & $128(30.40)$ & & 421 \\
\hline
\end{tabular}




\subsection{Imaging Data}

The patients included 306 cases of cerebral infraction (CI) (72.68\%) and 115 cases of intracerebral hemorrhage (ICH) (27.32\%). The distributions of aphasic types with different age and sex were summarized in Table 2 and Table 3.

The distribution of types of aphasia had no significant difference among the different type of stroke groups $\left(\chi^{2}\right.$ $=13.23, P=0.067)$, different age sub-groups $\left(\chi^{2}=14.94, P=0.382\right)$ and different sex group $\left(\chi^{2}=13.84, P=\right.$ 0.054). Broca's and Wernicke's aphasia were most common in CI patients. However, among ICH patients, the most common types were Broca's, anomic and global aphasia. Except for the females with ICH (may be caused by the small sample size), the above pattern could also be observed in different types of stroke, age and sex sub-groups.

\section{Discussion}

Aphasia refers to the loss or impairment of language communication skills owing to the brain disease [3] [13]. With the increasing morbidity of cerebrovascular disease in China, aphasia, as one of the common complications, is paid more and more attention. With Benson Classification [14] as reference, combined with clinical requirement, aphasia of Chinese can be divided into the following types based on the fluency, comprehension and naming ability: non-fluent aphasia (including Broca's aphasia, transcortical motor aphasia, global aphasia and transcortical combined aphasia) and fluent aphasia (including anomic aphasia, conductive aphasia, Wernicke's

Table 2. The distribution of the types of aphasia of ICH patients in different age groups [n (\%)].

\begin{tabular}{cccccccc} 
& \multicolumn{2}{c}{ Youth $(\leq 40 \mathrm{y})$} & \multicolumn{2}{c}{ Middle-aged $(41-65 \mathrm{y})$} & \multicolumn{2}{c}{ Elderly $(>65 \mathrm{y})$} & Total \\
\cline { 2 - 6 } & Male & Female & Male & Female & Male & Female & \\
\hline Broca's & $7(38.89)$ & $0(0.00)$ & $10(23.81)$ & $4(16.00)$ & $3(20.00)$ & $1(11.11)$ & $25(21.74)$ \\
Wernicke’s & $3(16.67)$ & $0(0.00)$ & $3(7.14)$ & $4(16.00)$ & $1(6.67)$ & $1(11.11)$ & $12(10.43)$ \\
Conductive & $1(5.56)$ & $1(16.67)$ & $1(2.38)$ & $3(12.00)$ & $0(0.00)$ & $0(0.00)$ & $6(5.22)$ \\
Transcortical sensory & $1(5.56)$ & $1(16.67)$ & $1(2.38)$ & $1(4.00)$ & $2(13.33)$ & $0(0.00)$ & $6(5.22)$ \\
Transcortical motor & $1(5.56)$ & $0(0.00)$ & $10(23.81)$ & $1(4.00)$ & $3(20.00)$ & $2(22.22)$ & $17(14.78)$ \\
Transcortical combined & $0(0.00)$ & $2(33.33)$ & $5(11.90)$ & $3(12.00)$ & $0(0.00)$ & $1(11.11)$ & $11(9.57)$ \\
Global & $3(16.67)$ & $1(16.67)$ & $9(21.43)$ & $6(24.00)$ & $4(26.67)$ & $1(11.11)$ & $24(20.87)$ \\
Anomic & $2(11.11)$ & $1(16.67)$ & $3(7.14)$ & $3(12.00)$ & $2(13.33)$ & $3(33.33)$ & $14(12.17)$ \\
\hline
\end{tabular}

Table 3. The distribution of the types of aphasia of CI patients in different age groups [n (\%)].

\begin{tabular}{|c|c|c|c|c|c|c|c|}
\hline & \multicolumn{2}{|c|}{ Youth $(\leq 40 \mathrm{y})$} & \multicolumn{2}{|c|}{ Middle-aged (41 - 65 y) } & \multicolumn{2}{|c|}{ Elderly (>65 y) } & \multirow{2}{*}{ Total } \\
\hline & Male & Female & Male & Male & Female & Male & \\
\hline Broca’s & $7(30.43)$ & $2(28.57)$ & $41(30.83)$ & 10 (28.57) & $17(27.42)$ & $14(30.43)$ & $91(29.74)$ \\
\hline Wernicke’s & $1(4.35)$ & $2(28.57)$ & $8(6.02)$ & $4(11.43)$ & $4(6.45)$ & $4(8.70)$ & $23(7.52)$ \\
\hline Conductive & $2(8.70)$ & $0(0.00)$ & $3(2.26)$ & $1(2.86)$ & $3(4.84)$ & $0(0.00)$ & $9(2.94)$ \\
\hline Transcortical sensory & $1(4.35)$ & $0(0.00)$ & $1(0.75)$ & $0(0.00)$ & $2(3.23)$ & $1(2.17)$ & $5(1.63)$ \\
\hline Transcortical motor & $1(4.35)$ & $1(14.29)$ & $26(19.55)$ & $6(17.14)$ & $9(14.52)$ & $3(6.52)$ & $46(15.03)$ \\
\hline Transcortical combined & $0(0.00)$ & $0(0.00)$ & $5(3.76)$ & $2(5.71)$ & $3(4.84)$ & $6(13.04)$ & $16(5.23)$ \\
\hline Global & $5(21.74)$ & $0(0.00)$ & $25(18.80)$ & $4(11.43)$ & $14(22.58)$ & $9(19.57)$ & $57(18.63)$ \\
\hline Anomic & $6(26.09)$ & $2(28.57)$ & $24(18.05)$ & $8(22.86)$ & $10(16.13)$ & $9(19.57)$ & $59(19.28)$ \\
\hline
\end{tabular}


aphasia, transcortical sensory aphasia and subcortical aphasia) [3]. Some foreign and domestic studies suggested that there were many factors affecting the types of post-stroke aphasia, such as lesion location, age, sex and stage of stroke. Some domestic scholars [15] thought the lesion location was the major factor influencing the aphasic type in acute phase of stroke, while other studies [16] showed that the association between the lesion location and the types of aphasia was not totally accorded with the classic aphasia models, so we could not completely rely on the lesions to determine the aphasic type. Recently, it's well acknowledged that age had the following characteristics of the effect on the types of aphasia: patients of Broca's aphasia were younger than that of Wernicke's aphasia, and this difference only can be seen in ischemic stroke. However, our study found that the age has no significant effect on aphasic types in both CI and ICH patients $\left(\chi^{2}=14.33, P=0.425 ; \chi^{2}=10.46, P=\right.$ $0.728, \alpha=0.05$ ). On the one hand, this result may be explained by the small sample size, and on the other hand, we didn't have a further analysis about the specific lesions, which may lead to the different results. In regard to gender, there were few relevant studies. Some [17] [18] thought male patients tended to suffered from Broca's aphasia and females tended from Wernicke's global or anomic aphasia. However, our study found that the Broca's aphasia was the most common type in both male and female. We also got some similar conclusion with other studies: sex had no significant effect on the types of aphasia. Some domestic researchers demonstrated that sex had effect on the severity and recovery of aphasia. That is, the degree of speech disorder in male patients was more worse and the recovery was slower than females [19]. The association between sex and aphasia can be explained by the difference of cerebral hemisphere structure and behavior [20]. During the fetal brain development, different sex hormones caused the different structure of language processing of brain in males and females, and recently the functional magnetic resonance imaging had confirmed this view [21] [22]. The language processing was governed by the bilateral cerebral hemisphere in females and by the unilateral hemisphere in males. When executing language tasks, activating areas in females were larger than males, the former located in bilateral and the latter located in unilateral.

Relevant clinical experiences and studies [23]-[25] showed that the incidence rate of stroke in males and females had remarkable difference in different ages. Male patients had a significantly higher level of morbidity of stroke and a younger average age of onset than females. For cerebral infraction, the peak ages of onset were 45 59 years old in males and 60 - 69 years old in females. Our study had gotten the similar result. To some degree, the higher level of morbidity of aphasia and stroke in males may be related with the unhealthy life styles in males and the brain and vascular protection of estrogen in females.

Note that the present study has some limitations. The data size of female patients was too small to draw a reliable and more scientific result. We failed to collect the AQ scores of most patients after recovery.

\section{Conclusion}

To be concluded, age, sex and stroke types have no significant influence on the types of aphasia. Male patients have a significantly higher level of morbidity of aphasia and younger average age of onset than females after stroke. Broca's aphasia is the most common type of aphasics in both male and female, followed by anomic and global aphasia.

\section{Acknowledgements}

This study was funded by Open Program of the National Key Laboratory of Cognitive Neuroscience and Learning (Beijing Normal University), National Social Science Foundation Program (11AZD119), National Social Science Foundation Key Program (11 \& ZD186), National Science Foundation of China (NSFC, 81371201), Beijing High-level Healthcare Technology development Plan (2011-3-024), National Key Technology Research and Development Program of the Ministry of Science and Technology of China (2013BAI09B03) and the project of Beijing Municipal Commission of Education (350011320061).

\section{References}

[1] Vidovic, M., Sinanovic, O., Sabaskic, L., et al. (2011) Incidence and Types of Speech Disorders in Stroke Patients. Acta Clinica Croatica, 50, 491-494.

[2] Kadojic, D., Bijelic, B.R., Radanovic, R., et al. (2012) Aphasia in Patients with Ischemic Stroke. Acta Clinica Croatica, 51, 221-225.

[3] Sinanovic, O., Mrkonjic, Z., Zukic, S., et al. (2011) Post-Stroke Language Disorders. Acta Clinica Croatica, 50, 79-94. 
[4] Dickey, L., Kagan, A., Lindsay, M.P., et al. (2010) Incidence and Profile of Inpatient Stroke-Induced Aphasia in Ontario, Canada. Archives Physical Medicine and Rehabilitation, 91, 196-202. http://dx.doi.org/10.1016/j.apmr.2009.09.020

[5] Gialanella, B., Bertolinelli, M., Lissi, M., et al. (2011) Predicting Outcome after Stroke: The Role of Aphasia. Disability and Rehabilitation, 33, 122-129. http://dx.doi.org/10.3109/09638288.2010.488712

[6] Miyashita, H. and Sakai, K.L. (2011) Localization of Language Function in the Brain. Brain and Nerve, 63, 13391345.

[7] Kang, E.K., Sohn, H.M., Han, M.K., et al. (2010) Severity of Post-Stroke Aphasia According to Aphasia Type and Lesion Location in Koreans. Journal of Korean Medical Science, 25, 123-127. http://dx.doi.org/10.3346/jkms.2010.25.1.123

[8] Otsuki, M. (2008) Aphasia in Practice-Recent Progress. Rinsho Shinkeigaku, 48, 853-856. http://dx.doi.org/10.5692/clinicalneurol.48.853

[9] Trudeau, N., Goulet, P. and Joanette, Y. (1993) Age and Type of Aphasia. Acta Neurologica Belgica, 93, $283-289$.

[10] Steinvil, Y., Ring, H. and Luz, Y. (1985) Type of Aphasia: Relationship to Age, Sex, Previous Risk Factors, and Outcome of Rehabilitation. Scandinavian Journal Rehabilitation Medicine Supplement, 12, 68-71.

[11] The Chinese Medical Association of the Fourth National Cerebrovascular Disease Conference (1996) Essentials of Diagnosis of All Kinds of Cerebrovascular Diseases. Chinese Journal of Neurology, 29, 379.

[12] Gao, S.R. (1992) Discussion about the Standardization of Aphasia Battery of Chinese. Journal of Clinical Neurology, 5, 193-195.

[13] Gao, S.R. (2006) Aphasia. 2nd Version, Peking University Medical Press, Beijing, 4-5.

[14] Obler, L.K., Albert, M.L., Benson, D.F. and Goodglass, H. (1978) Aphasia Type and Aging. Brain and Language, 6, 318-322. http://dx.doi.org/10.1016/0093-934X(78)90065-2

[15] Yang, Z.H., Zhao, X.Q., Wang, C.X., Chen, H.Y. and Zhang, Y.M. (2008) Neuroanatomic Correlation of the PostStroke Aphasias Studied with Imaging. Neurological Research, 30, 356-360. http://dx.doi.org/10.1179/174313208X300332

[16] Zhang, Y.M., Wang, Y.J., Zhou, Y., et al. (2005) The Clinical Study of the Association between the Types of Aphasia and Lesion Localization. Chinese Journal of Rehabilitation Medicine, 20, 352-353.

[17] Hier, D.B., Yoon, W.B., Mohr, J.P., Price, T.R. and Wolf, P.A. (1994) Gender and Aphasia in the Stroke Data Bank. Brain and Language, 47, 155-167. http://dx.doi.org/10.1006/brln.1994.1046

[18] De Renzi, E., Faqlioni, P. and Ferrari, P. (1980) The Influence of Sex and Age on the Incidence and Type of Aphasia. Cortex, 16, 627-630. http://dx.doi.org/10.1016/S0010-9452(80)80010-4

[19] Chen, Y. and Li, Y.S. (2009) The Effect of Sex on the Post-Stroke Aphasia. Journal of Shanghai Jiao Tong University (Medical Science), 29, 978-981.

[20] Zhang, Y.M. and Wang, Y.J. (2004) Relevant Factors about the Types of Aphasia. Chinese Journal of Rehabilitation Theory and Practice, 10, 241-242.

[21] Bitan, T., Lifshitz, A., Breznitz, Z. and Booth, J.R. (2010) Bidirectional Connectivity between Hemispheres Occurs at Multiple Levels in Language Processing but Depends on Sex. The Journal of Neuroscience, 30, 11576-11585. http://dx.doi.org/10.1523/JNEUROSCI.1245-10.2010

[22] Clements-Stephens, A.M., Rimrodt, S.L. and Cutting, L.E. (2009) Developmental Sex Differences in Basic Visuospatial Processing: Differences in Strategy Use? Neuroscience Letters, 449, 155-160. http://dx.doi.org/10.1016/j.neulet.2008.10.094

[23] Mu, X.D. (2012) Analysis of Gender Differences in First-Ever Ischemic Stroke Imaging and Epidemiology. Hebei Medical University, Shijiazhuang.

[24] Geng, Y.Y. (2011) Gender Differences of Clinical Features and Prognosis of Primary Cerebral Hemorrhage. Wenzhou Medical College, Wenzhou.

[25] Appelros, P., Stegmayr, B. and Terént, A. (2009) Sex Differences in Stroke Epidemiology: A Systematic Review. Stroke, 40, 1082-1090. http://dx.doi.org/10.1161/STROKEAHA.108.540781 
Scientific Research Publishing (SCIRP) is one of the largest Open Access journal publishers. It is currently publishing more than 200 open access, online, peer-reviewed journals covering a wide range of academic disciplines. SCIRP serves the worldwide academic communities and contributes to the progress and application of science with its publication.

Other selected journals from SCIRP are listed as below. Submit your manuscript to us via either submit@scirp.org or Online Submission Portal.
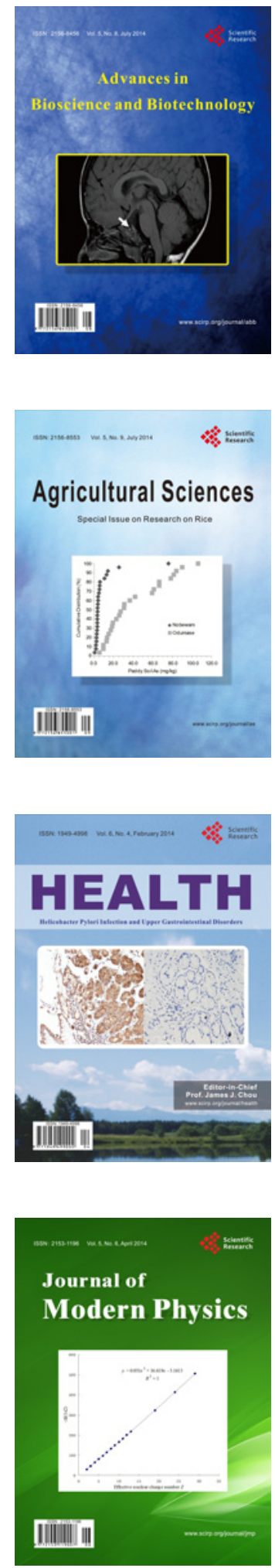
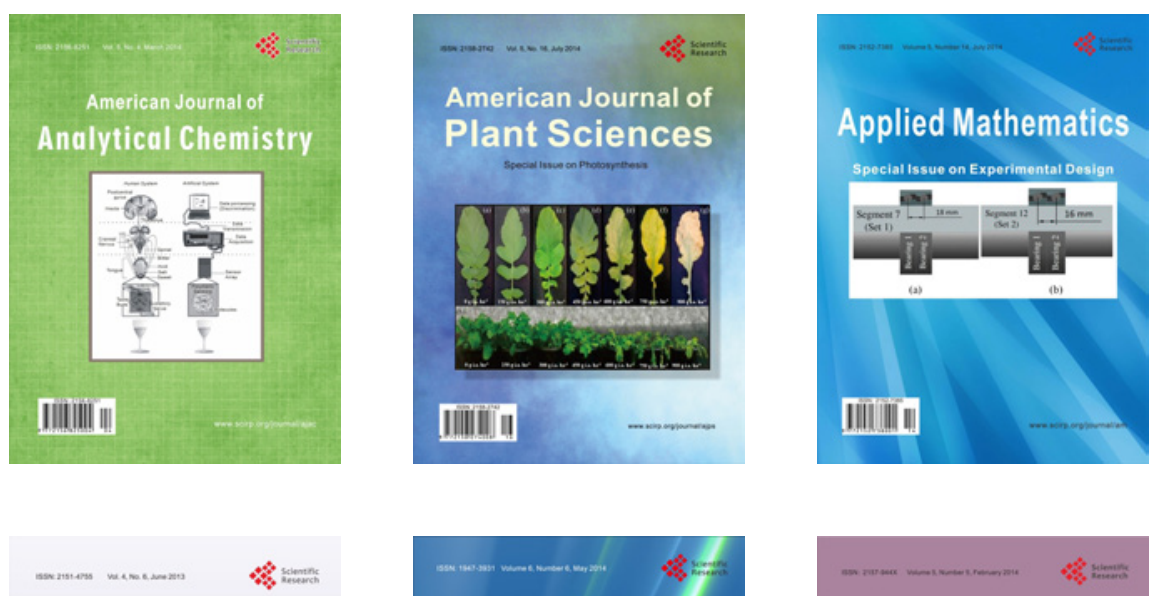

Creative Education
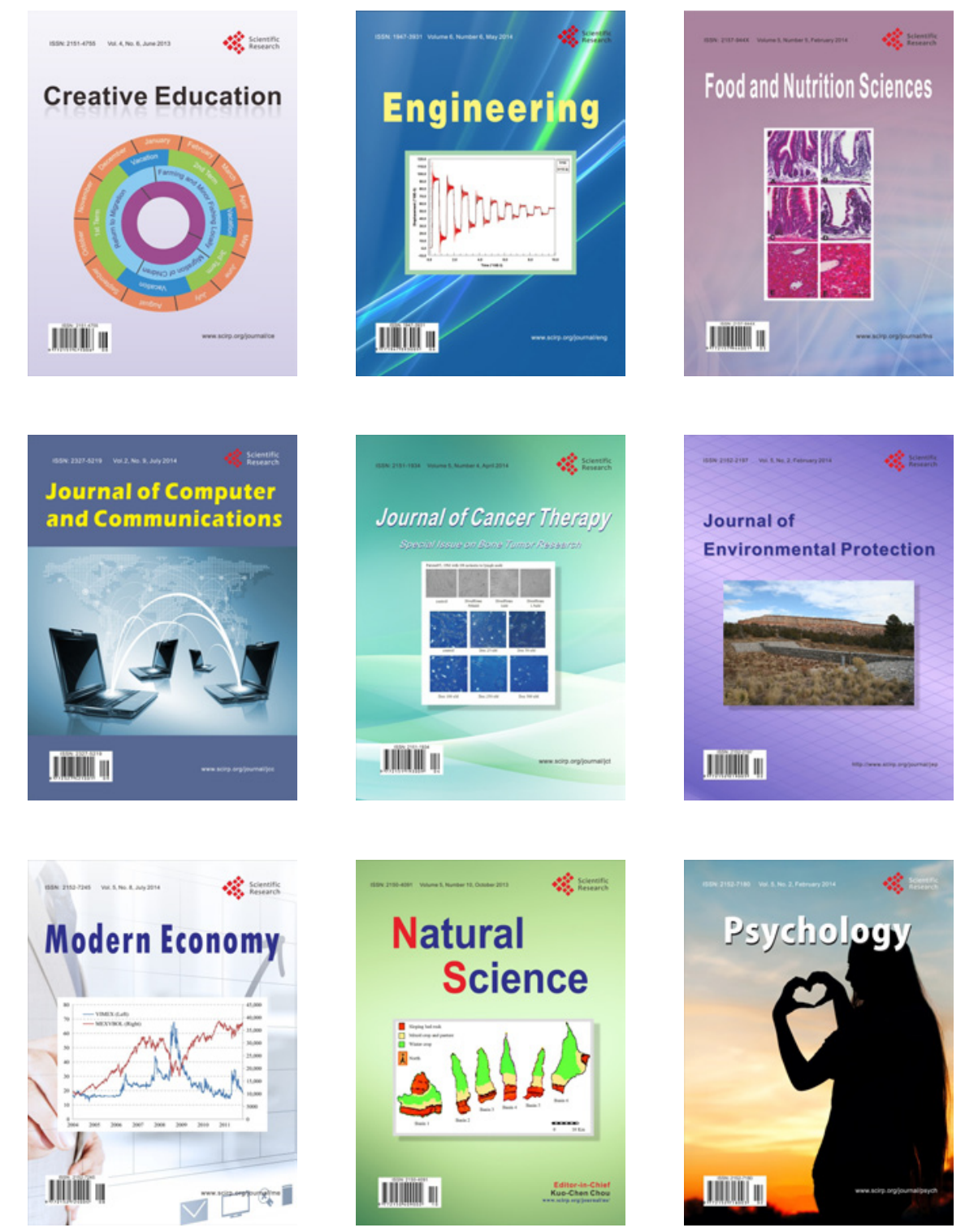